\title{
A curvature sensor based on twisted single-mode-multimode- single-mode hybrid optical fiber structure
}

\author{
Ke Tian \\ Harbin Engineering University \\ Yifan Xin \\ Harbin Engineering University \\ Wenlei Yang \\ Harbin Engineering University
}

See next page for additional authors

Follow this and additional works at: https://arrow.tudublin.ie/prcart

Part of the Electrical and Computer Engineering Commons

\section{Recommended Citation}

K. Tian et al., "A Curvature Sensor Based on Twisted Single-Mode-Multimode-Single-Mode Hybrid Optical Fiber Structure," in Journal of Lightwave Technology, vol. 35, no. 9, pp. 1725-1731, 1 May1, 2017, doi:

10.1109/JLT.2017.2650941.

This Article is brought to you for free and open access by the Photonics Research Centre at ARROW@TU Dublin. It has been accepted for inclusion in Articles by an authorized administrator of ARROW@TU Dublin. For more information, please contact arrow.admin@tudublin.ie, aisling.coyne@tudublin.ie,gerard.connolly@tudublin.ie.

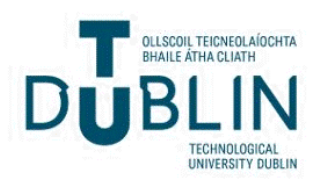


Authors

Ke Tian, Yifan Xin, Wenlei Yang, Tao Geng, Jing Ren, Ya-Xian Fan, Gerald Farrell, Elfed Lewis, and Pengfei Wang

This article is available at ARROW@TU Dublin: https://arrow.tudublin.ie/prcart/30 
See discussions, stats, and author profiles for this publication at: https://www.researchgate.net/publication/312207227

\section{A Curvature Sensor Based on Twisted Single-Mode-Multimode-Single-Mode Hybrid Optical Fiber Structure}

Article in Journal of Lightwave Technology · January 2017

DOI: 10.1109/JLT.2017.2650941

\section{CITATIONS}

36

9 authors, including:

Harbin Engineering University

37 PUBLICATIONS 382 CITATIONS

SEE PROFILE

(3)

Harbin Engineering University

131 PUBLICATIONS 1,807 CITATIONS

SEE PROFILE

Some of the authors of this publication are also working on these related projects:
READS

308

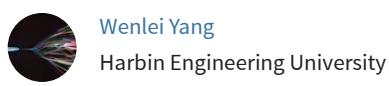

39 PUBLICATIONS 365 CITATIONS

SEE PROFILE

Ya-Xian Fan

Guilin University of Electronic Technology

99 PUBLICATIONS 1,080 CITATIONS

SEE PROFILE

Project Optical Fibre Sensor for ultra low level ethanol concentration measurement in bio-fuel processing applications View project

Project optic fiber View project 


\title{
A Curvature Sensor Based on Twisted Single-Mode-Multimode-Single-Mode Hybrid Optical Fiber Structure
}

\author{
Ke Tian, Yifan Xin, Wenlei Yang, Tao Geng, Jing Ren, Ya-Xian Fan, Gerald Farrell, \\ Elfed Lewis, Senior Member, IEEE, and Pengfei Wang
}

\begin{abstract}
An optical fiber curvature sensor based on a twisted multimode fiber (MMF) sandwiched between two single-mode fibers (SMF) is proposed and investigated theoretically and experimentally. The measured transmission spectrum exhibits good agreement with theoretical predictions. Compared with a traditional single-mode-multimode-single-mode fiber structure sensor, the proposed configuration offers a higher average curvature sensitivity of $-2.42 \mathrm{~nm} / \mathrm{m}^{-1}$ over a curvature measurement range of 0-1.7390 $\mathrm{m}^{-1}$ and $-7.09 \mathrm{~dB} / \mathrm{m}^{-1}$ at an operating wavelength of $1537 \mathrm{~nm}$. The temperature sensitivity of this sensor has been determined as $0.01 \mathrm{~nm} /{ }^{\circ} \mathrm{C}$ over a wavelength range of $1535-1550 \mathrm{~nm}$ and circa $0.007 \mathrm{~dB} /{ }^{\circ} \mathrm{C}$ at the wavelength of $1537 \mathrm{~nm}$, over a measured temperature range of $21-121^{\circ} \mathrm{C}$.
\end{abstract}

Index Terms-Curvature sensor, Mach-Zehnder interferometer, multimode interference, temperature dependence.

\section{INTRODUCTION}

$\mathbf{T}$ HE well-established hybrid single-mode-multimodesingle-mode (SMS) fibre structure has been widely investigated and exhibits a number of advantages, such as compact structure, low insertion loss and ease of fabrication. A comprehensive theoretical description of the SMS fibre structure has been developed and is described in [1]. The operating principle of the hybrid SMS fibre is based on multimode interference excited in the multimode fibre section, which is also the underlying operating principle of the SMS

Manuscript received October 14, 2016; revised December 20, 2016; accepted January 4, 2017. Date of publication January 9, 2017; date of current version April 20, 2017. This work was supported by the 111 project (B13015) at the Harbin Engineering University, the Fundamental Research Funds of the Central University and the Harbin Engineering University, and by the National Natural Science Foundation of China under Grant 61575050. (Corresponding author: Pengfei Wang.)

K. Tian, Y. Xin, W. Yang, T. Geng, J. Ren, and Y. Fan are with the Key Laboratory of In-fiber Integrated Optics of Ministry of Education, College of Science, Harbin Engineering University, Harbin 150001, China (e-mail: ketian@hrbeu.edu.cn; xinyifan@hrbeu.edu.cn; ywl-@hotmail.com; gengtao_hit_oe@126.com; ren.jing@hrbeu.edu.cn; yxfan@ hrbeu.edu.cn).

P. Wang and G. Farrell are with the Key Laboratory of In-fiber Integrated Optics of Ministry of Education, College of Science, Harbin Engineering University, Harbin 150001, China, and also with the Photonics Research Centre, Dublin Institute of Technology, Dublin 8, Ireland (e-mail: pengfei.wang @ dit.ie; gerald.farrell@dit.ie).

E. Lewis is with the Optical Fibre Sensors Research Centre, Department of Electronic and Computer Engineering, University of Limerick, Limerick V94 T9PX, Ireland (e-mail: Elfed.lewis@ul.ie).

Color versions of one or more of the figures in this paper are available online at http://ieeexplore.ieee.org.

Digital Object Identifier 10.1109/JLT.2017.2650941 fibre structures. The hybrid SMS fibre has been demonstrated for a wide range of applications in telecommunication and fibre optic sensing, including edge filters [2], [3], bandpass filters [4], [5] as well as a variety of sensors for measurands including temperature [6], strain [7], curvature [8] and refractive index (RI) [9], [10]. Additionally, due to the relative ease of light coupling and interconnection of the wide cored SMS fibre structure, it can be easily combined with other fibre components, e.g. It can be fusion spliced with single mode fibres which include a fibre Bragg grating (FBG) to achieve multi-parameter measurement [11], [12].

Optical fibre based curvature sensors have been widely investigated for applications in industry, medicine and structural healthy monitoring. To date several types of optical fibre based curvature sensors have been proposed. The most common approaches include on the use of long-period gratings (LPGs) [13], [14], FBGs [15], [16], core-offset fibre [17] and SMS fibre structures [18]. SMS fibre curvature sensors offer advantages such as low cost and ease of fabrication, without the use of expensive masks, compared with the other existing technologies used for curvature measurement based on optical fibre.

To date improvement in performance of the traditional SMS fibre structure, has been achieved using a range of postfabrication methods. Examples include chemically removing the cladding of multimode fibre section for increasing the sensitivity of refractive index measurement [9] and single-stretching [10] or multi-tapers [19] on the multimode fibre section for generating an enhanced evanescent wave, hence significantly increasing the sensitivity of the hybrid SMS fibre structure. However, both of the two fabrication methods increase the fabrication difficulty and the resulting mechanical instability may lead to significant breakage risk during use. It is desirable to further improve the performance of the SMS fibre structure in terms of achieving a high sensitivity on curvature monitoring while retaining good mechanical stability and robustness. In this paper, a novel singlemode-twisted multimode-singlemode (STMS) fibre structure is described and investigated. The schematic configuration of the proposed STMS fibre structure is shown in Fig. 1. The hybrid fibre configuration under consideration consists of an input single-mode fibre (SMF), a multimode fibre (MMF) with two twist points and an output SMF. Note that both twist sections are permanently generated using a combined filament heating and twisting process. The twist changes not only the 


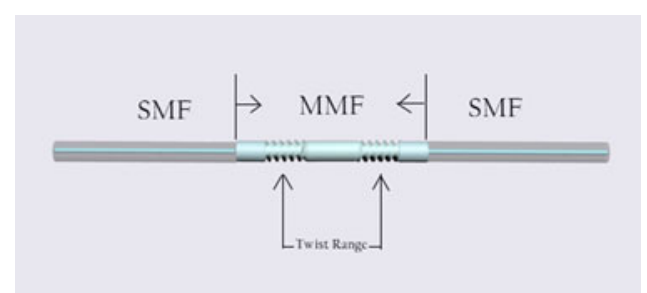

Fig. 1. Schematic of the STMS fibre structure with two twisted points.

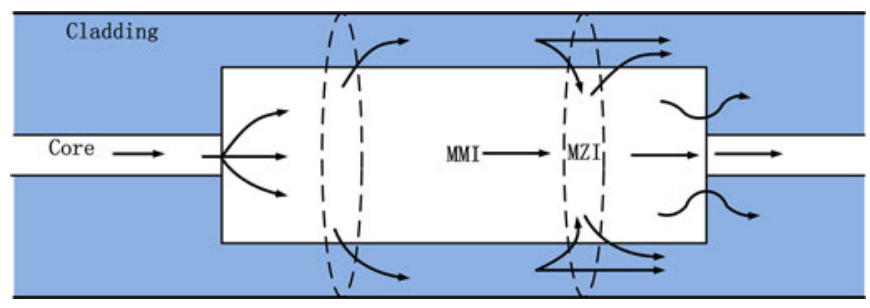

Fig. 2. Schematic diagram of STMS fibre (dotted curve express twisted area).

shear stress but also the local refraction indices of the MMF. The multimode interference generated within the multimode section is significantly effected by both twist sections on the MMF [20]. Additionally, a Mach-Zehnder interference induced by the two twist sections is realised in this fibre structure.

\section{THEORETICAL ANALYSIS}

A schematic diagram is illustrated in Fig. 2 to explain the light propagation and interference signal development within the STMS fibre structure. The input signal launched to the MMF section can be treated as the fundamental mode of the SMF, the eigenmodes of $\mathrm{LP}_{0 \mathrm{~m}}$ of the MMF can be excited provided that the central axes of the fibre cores of the SMF and MMF are perfectly aligned, in which case strong mode interference occurs within the MMF section. When the two twisted sections are introduced on the MMF section, the excited $\mathrm{LP}_{0 \mathrm{~m}}$ modes will be partially coupled to the high-order cladding modes at the first twisted point and this increases the fraction of power in the evanescent field within the region of MMF cladding. Fig. 2 illustrates the mechanism through which the MMF high-order modes leak from the MMF core into the MMF cladding at the first twist point, traveling in the MMF cladding region between the two twisted points, and coupled back to the fibre core at the second twisted section. As a result, interference occurs between the cladding and core modes. Therefore the two twist points form a fibre Mach-Zehnder Interferometer (MZI) structure. For the light coupled from the MMF into the output SMF, a portion of the light enters the SMF core while the remainder is coupled into the output SMF cladding (Fig. 2).

In the well-established mode theory describing the process of high-order mode coupling in the multimode fibre [20], the coupling status can be assumed to be "statistically isotropic" [21], [22]. This assumption saves computational time and improves the numerical computation efficiency. However, for the twisted fibre case, the magnitude and spatial scale of the high-order modes coupling are significantly different from the straight case. Since the step-index MMF is an isotropic and symmetrical structure, only the coupling that occurs among the same manifold modes (intra-manifold coupling) is considered. Under this assumption, the coupling rate $K$ can be calculated by the integrals:

$$
K_{u, v}=\frac{\omega}{4} \int_{0}^{\infty} \int_{0}^{2 \pi} r E_{u}^{*} \tilde{\varepsilon} E_{v} d \varphi d r=K_{v, u}^{*}
$$

where $u$ and $v$ are the different mode indices and $E_{u}$ and $E_{v}$ are their corresponding electric fields value, $r$ and $\Phi$ are the coordinates of a cylindrical reference frame, $\tilde{\varepsilon}$ is a $3 \times 3$ matrix representing the permittivity tensor. When the multimode fibre is twisted, the axial and shear stresses of the fibre are radically altered, $\tilde{\varepsilon}$ is varied by a perturbation due to the elasto-optical effect:

$$
\tilde{\varepsilon}=g \tau \varepsilon_{0} n_{\mathrm{av}} r\left(\begin{array}{ccc}
0 & 0 & -\sin \varphi \\
0 & 0 & \cos \varphi \\
-\sin \varphi & \cos \varphi & 0
\end{array}\right),
$$

where $g$ is the elasto-optic coefficient, $\tau$ is twist-rate (twist applied per unit length), and $n_{\text {av }}$ is the mean refractive index. From equations (1) and (2) it is clear that two linearly polarized $(L P)$ modes such as $L P_{m, n}$ and $L P_{p, q}$ can only be coupled when $m=p$ or $|m-p|=2$ (in any other case, the matrix yields zero).

The twist in the sensing fibre not only induces a change of the elasto-optic coefficient, but also a geometrical rotation of the MMF itself. Such rotation also results in a perturbation of the high-order modes coupling and affect the coupling matrix. If it is assumed that the final coupling matrix is $\mathrm{K}^{\prime}$, let A be the twist-induced coupling matrix due to elasto-optic effect which has been previously discussed in equation (2) presented above. B is the coupling matrix which is a perturbation not induced by twist. When the fibre is twisted, matrix $A$ is induced by twist, and for the matrix $\mathrm{B}$, the geometrical rotation transforms the coupling matrix $\mathrm{B}$ to $B^{\prime}=R(\theta) B R^{T}(\theta)$, where $\theta$ is rotation angle, and it can be calculated by straightforward integration: $\theta(x)=\int_{0}^{x} \tau(u) d u$, note that $\tau$ is the twist-rate of fibre and $R(\theta)=\operatorname{diag}\left(R_{i 1}(\theta), R_{i 2}(\theta), \ldots\right)$ is a block-diagonal matrix [23]. The corresponding $L P$ manifold can be represented by each block as:

$R_{0}=\left(\begin{array}{cc}\cos \theta & -\sin \theta \\ \sin \theta & \cos \theta\end{array}\right), R_{n}=\left(\begin{array}{cc}R_{0} & 0 \\ 0 & R_{0}\end{array}\right)\left(\begin{array}{cc}I \cos n \theta & -I \sin n \theta \\ I \sin \theta & I \cos n \theta\end{array}\right)$,

where $R_{0}$ corresponds to $L P_{0, q}$ manifolds, $R_{n}(\theta)$ corresponds to $L P_{n, q}$ modes. The coupling matrix is ultimately determined as $\mathrm{K}^{\prime}=\mathrm{A}+\mathrm{B}^{\prime}$.

Based on the above theoretical analysis of the mode coupling in the case of the STMS fibre, a commercial software BeamPROP (Rsoft, Pasadena CA USA) based on a 3-dimensional finite difference beam propagating method (FD-BPM) has been used to calculate the optical intensity distribution with light propagation as shown in Fig. 3. The mesh size on X and Y direction is $0.1 \mu \mathrm{m}$ and the mesh size on the propagation direction $\mathrm{Z}$ is $1 \mu \mathrm{m}$, a perfectly matched layer condition has been adopted as the boundary condition in the model. Fig. 3 shows that the 


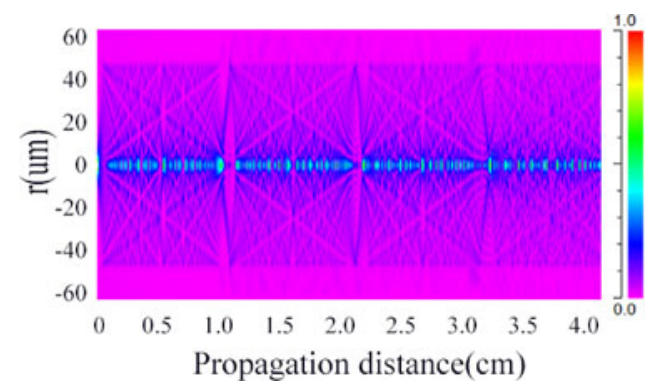

Fig. 3. Simulated amplitude of Optical fields of the STMS fibre structure at a wavelength of $1550 \mathrm{~nm}$ using a FD-BPM.

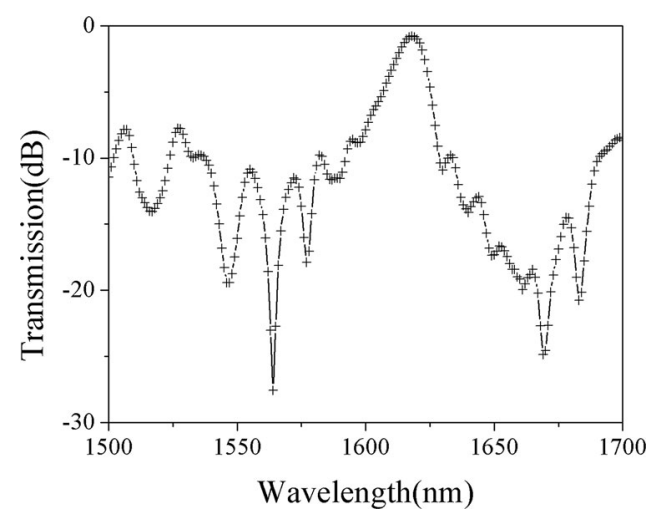

Fig. 4. Simulated transmission spectrum of the STMS fibre structures.

high-order MMF core modes leak out from the MMF core into the MMF cladding at the first twist point, travel in the MMF cladding region between the two twist points, and coupled back to the fiber core at the second twisted section. Fig. 4 shows the simulated transmission spectrum of the STMS fibre structure with a length of $20 \mathrm{~mm}$ between the two twisted points which has been fabricated using the filament heater. It is clear that the larger dips induced by mode interference occur over the entire transmission spectrum and these dips can be used for sensing applications.

\section{FABRICATION OF STMS FIBRE SAMPLES}

The entire fabrication process can be divided into four steps as follows: 1) Firstly, an SMS fibre structure was prepared by using a commercial fusion splicer (Fujikura 62S, Japan). The SMF and MMF used in the experiments were SMF28 and AFS105/125Y, respectively. The entire length of the MMF used was $41.5 \mathrm{~mm}$; 2) Secondly, one of the SMFs was mounted on the fibre holder and the other was mounted on the rotatable fibre holder, as illustrated in Fig. 5. Then two twisted sections were selected on the MMF section, the distance between the end of the MMF and the beginning of the twisted section was circa $20 \mathrm{~mm}$; 3) The filament heater heated the MMF and the SMS fibre structure was continuously rotated using the rotatable fibre holder, thus creating the first twist point; 4) The filament heater was relocated to a new position using a linear translation stage and the MMF was again heated to create the second twist point. The

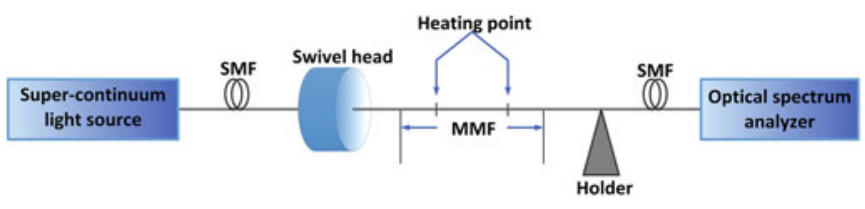

Fig. 5. Experimental setup for twist SMS fibre.

TABLE I

EXPERIMENTAL PARAMETERS FOR FABRICATION OF STMS FIBER STRUCTURE

\begin{tabular}{lcc}
\hline \hline Parameter & Data & Unit \\
\hline Heating Temperature & $1300 \pm 20$ & ${ }^{\circ} \mathrm{C}$ \\
Heating Length & 1 & $\mathrm{~mm}$ \\
Speed of Swivel & $\pi / 12$ & $\mathrm{rad} / \mathrm{s}$ \\
Number of Twisting Turns & 1.5 & $\mathrm{~N}$ \\
Distance Between Swivel Head and Holder & 2400 & $\mathrm{~mm}$ \\
\hline \hline
\end{tabular}

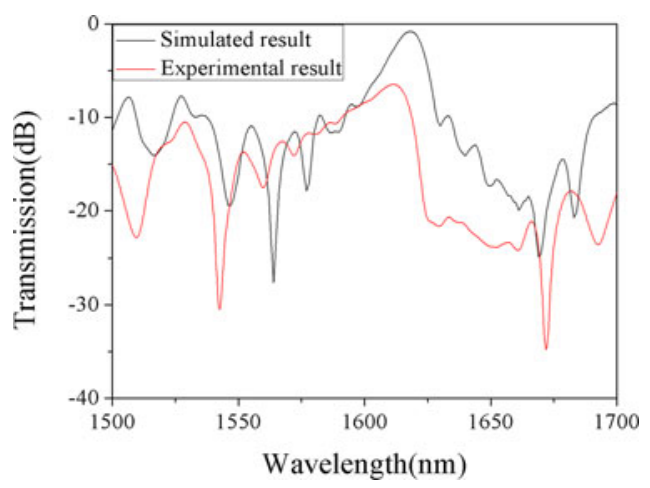

Fig. 6. Simulated data and experimental results of STMS fibre.

transmission spectra of the STMS fibre structure were analyzed on-line during fabrication using a broadband LED source and a high-resolution $(20 \mathrm{pm})$ optical spectrum analyzer (YOKOGAWA AQ6370D, operation wavelength range: $600-1700 \mathrm{~nm}$ ). The twisting process parameters, i.e. the temperature of filament heater, the heating length, the relative angular speed and the twisting turns were carefully optimized in order to achieve minimal transmission loss. The experimental parameters used in our experiment are shown in Table I. Fig. 5 shows the experimental setup for the fabrication of the STMS fibre structure. Fig. 6 presents the simulated and measured transmission spectra of the STMS fibre structures following the twisting process. From Fig. 6 it is clear that the measured results exhibit a general agreement with the theoretical predictions. The discrepancy between the theoretical predictions and measured results could be induced by deformities shape of the twisted sections on the MMF, the manufacturing tolerances on refractive indices and physical dimensions of the MMF fibre used in the experiments and approximations made during the simulation, e.g. transmission for a twisted MMF includes some approximations in the FD-BPM based model. Also the twist effect has changed the interference status of the high-order modes in the MMF section. 


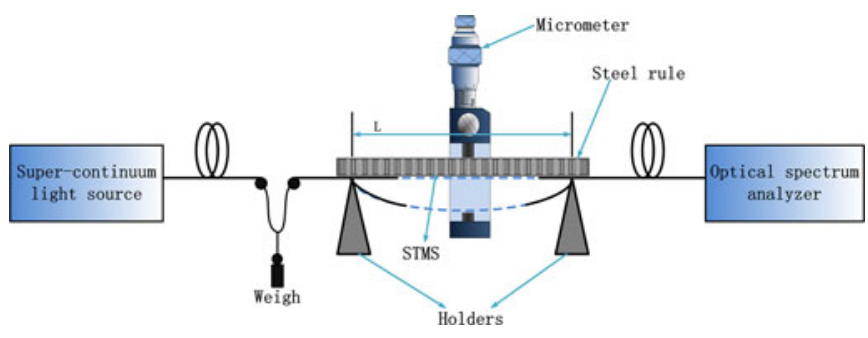

Fig. 7. Schematic diagram of the bend measurement setup.

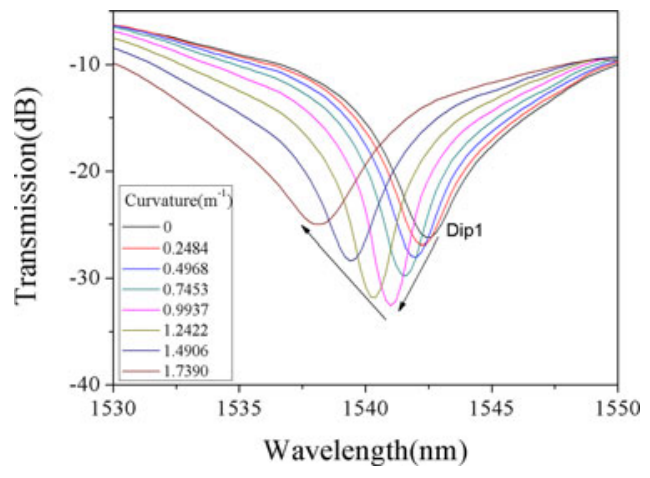

Fig. 8. Transmission spectral responses as a functions of different curvature.

\section{Curvature Measurements And Discussion}

The experimental setup for performing the curvature measurement is illustrated in Fig. 7. A super-continuum light source (SLS) was used as the input light. The output light signal was detected using an optical spectrum analyzer. The STMS fibre structure was placed at the center of two holders. One end of the STMS structure region was clamped using a holder and the other end was not fixed but was attached to a $5 \mathrm{~g}$-weight in order to prevent fibre breakage due to excessive stress. The distance between the two holders (L) was set to $139 \mathrm{~mm}$. This could be accurately determined using a steel rule pressed onto the fibre between the two holders. Above the steel rule, a micrometer caliper was used to change the radius of curvature by adjusting the screw gauge and the resulting bending displacement is d. The curvature of the STMS fibre structure can be determined using the formula $\rho=12 \mathrm{~d} / \mathrm{L}^{2}$. Fig. 8 shows the spectral response changes in curvature. In Fig. 8 it is clear that there is a distinct blue shift in the transmission spectrum at dip1 $(1542.4 \mathrm{~nm})$ when the curvature increases from 0 to 1.7390 $\mathrm{m}^{-1}$. The minimum $\mathrm{dB}$ transmission value of dip1 decreases within the curvature range $0-0.9937 \mathrm{~m}^{-1}$, and increases within curvature range $0.9937-1.7390 \mathrm{~m}^{-1}$. Fig. 9 represents the polynomial fit of the curvature sensing characteristic of dip1. A linear regression analysis of the dip wavelength position in Fig. 9(a) shows that within the curvature range of $0-1.7390 \mathrm{~m}^{-1}$, the average wavelength-curvature of dip 1 is $-2.42 \mathrm{~nm} / \mathrm{m}^{-1}$ with a regression coefficient value $\left(\mathrm{R}^{2}\right)$ of 0.9972 .

If viewed at a single wavelength, the shift of the interference dip means that the transmission (in $\mathrm{dB}$ ) is a strong function of curvature. For example for a wavelength of $1537 \mathrm{~nm}$,

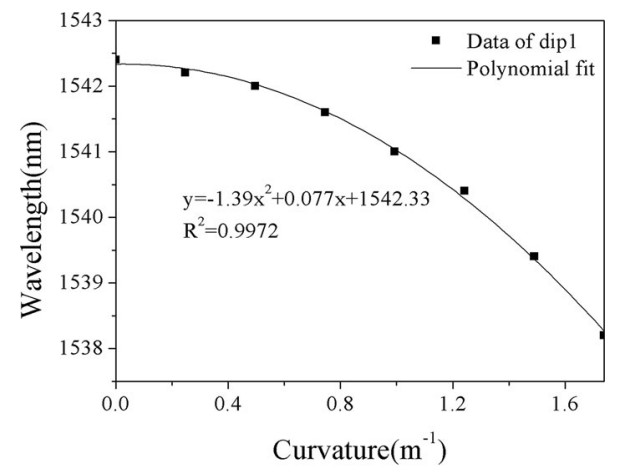

(a)

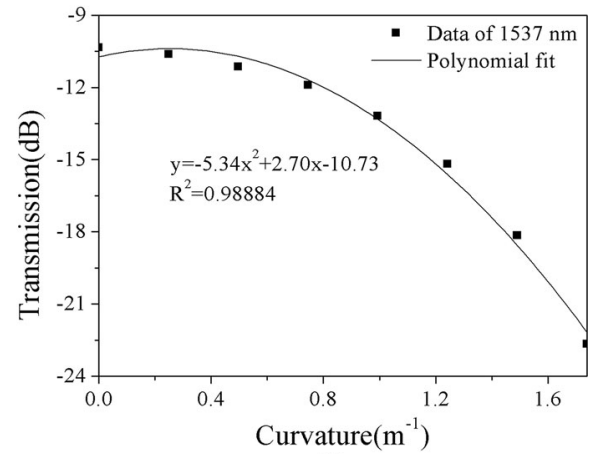

(b)

Fig. 9. Curvature responses of dip 1 (a) wavelength-curvature; (b) intensitycurvature (at $1537 \mathrm{~nm}$ ).

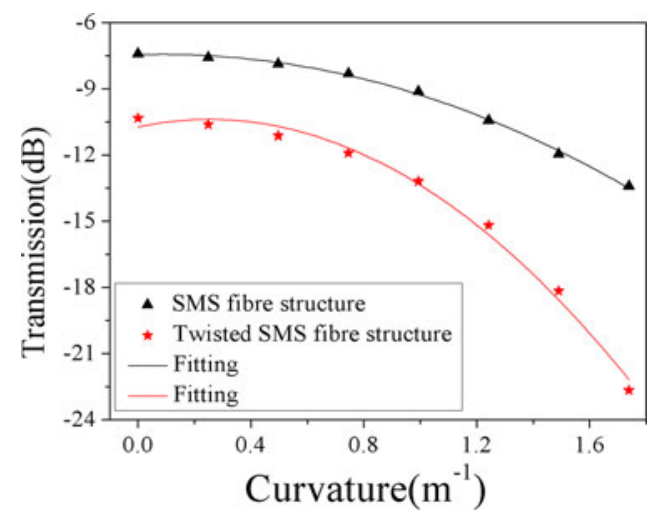

Fig. 10. Transmission changes of the traditional SMS fibre (triangle points fitted by a black line) and the proposed STMS fibre structure (star points fitted by a red line) as a function of different curvature.

the change in transmission for a curvature change between 0 and $1.7390 \mathrm{~m}^{-1}$ is $-12.33 \mathrm{~dB}$. Fig. 9(b) shows the intensitycurvature characteristic of $1537 \mathrm{~nm}$, within the curvature range of $0-1.7390 \mathrm{~m}^{-1}$. The average intensity-curvature sensitivities is $-7.09 \mathrm{~dB} / \mathrm{m}^{-1}$ with a regression coefficient value $\mathrm{R}^{2}$ of 0.9888 . To better compare with the performance of the curvature sensing of a traditional SMS fibre structure, the measured transmission changes of both the SMS and the STMS fibre structure are shown in Fig. 10. Fig 10 clearly shows that the curvature sensitivity of the STMS fibre structure is larger than that of the traditional SMS fibre structure within the curvature range of $0-1.7390 \mathrm{~m}^{-1}$, the multimode fibre length of SMS fibre structure is about 41 $\mathrm{mm}$ and the average sensitivity of curvature is $-3.45 \mathrm{~dB} / \mathrm{m}^{-1}$. 
TABLE II

Comparison of the Fibre CuRvature Sensors Developed to Date

\begin{tabular}{|c|c|c|c|}
\hline $\begin{array}{l}\text { Sensitivity of } \\
\text { Curvature }\end{array}$ & Linearity & Configuration & References \\
\hline $\begin{array}{l}8.7 \pm 0.1 \mathrm{~nm} / \mathrm{m}^{-1} \\
\text { (substantial } \\
\text { sensitivity) }\end{array}$ & not given & SMS fibre strcture & [8] \\
\hline Not calculated & not given & LPG & [13] \\
\hline Not calculated & not given & $\begin{array}{l}\text { All-fiber } \\
\text { Mach-Zehnder } \\
\text { combined with a } \\
\text { LPG }\end{array}$ & [14] \\
\hline $8.9 \mathrm{pm} / \mathrm{m}^{-1}$ & not given & $\begin{array}{l}\text { FBG in multicore } \\
\text { fibre }\end{array}$ & [15] \\
\hline Not calculated & not given & $\begin{array}{l}\text { FBG misaligned } \\
\text { with a SMF }\end{array}$ & [16] \\
\hline$-22.947 \mathrm{~nm} / \mathrm{m}^{-1}$ & 0.9903 & $\begin{array}{l}\text { SMFs using } \\
\text { core-offset splicing }\end{array}$ & [17] \\
\hline $\begin{array}{l}130.37 \mathrm{~dB} / \mathrm{m}^{-1} \\
\text { (maximum } \\
\text { sensitivity, curvature } \\
\text { range: } 0-0.3 \mathrm{~m}^{-1} \text { ) }\end{array}$ & not given & SMS fibre structure & [18] \\
\hline $\begin{array}{l}2.24 \mathrm{~nm} / \mathrm{m}^{-1} \text { and } \\
7.09 \mathrm{~dB} / \mathrm{m}^{-1} \\
\text { (average sensitivity) }\end{array}$ & $\begin{array}{c}0.9972 \text { and } \\
0.9888\end{array}$ & STMS fibre structure & Our work \\
\hline
\end{tabular}

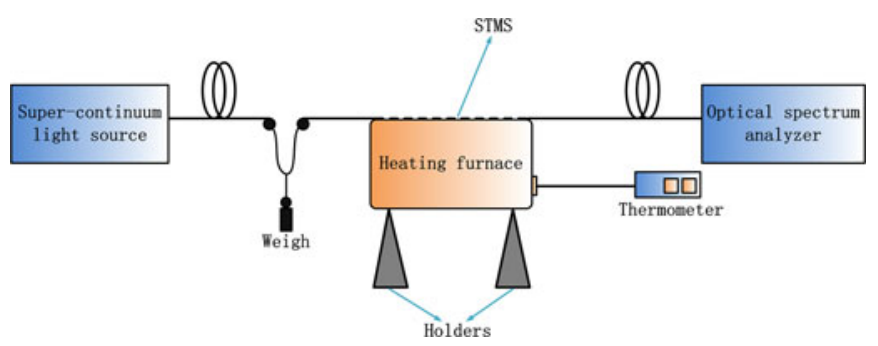

Fig. 11. Experimental setup for the temperature test.

During the experiments, no well-defined relationship between the length of the twisted section and the curvature sensitivity of the proposed sensor has been found, therefore here we have just presented the results of the twisted SMS fibres with a $2 \mathrm{~cm}$ long twist section with a maximal curvature sensitivity in the work.

In order to better compare with the sensitivity of the different fibre optic curvature sensors, Table II is provided for a direct comparison. Apart from the direct advantages of high sensitivity, the advantages such as ease of fabrication allows the possibility of new fibre optic sensor designs with a compact size, thereby reducing the size of the sensor, along with a low cost. As previously mentioned, the twisting process on the multimode fibre section has improved the sensing performance of the original SMS fibre structure significantly.

The temperature dependence of the proposed fibre curvature sensor was also studied, since temperature effects normally have a significant influence on the performance of a fibre optic sensor. To experimentally study the temperature dependence of the hybrid STMS fibre structure, the sample was placed in a heating furnace as shown in Fig. 11. The temperature of the heating furnace was controlled using a temperature controller. An additional handheld thermometer was used to confirm the temperature changes in the furnace. For the purpose of this experiment the ambient temperature inside the furnace was strictly fixed at

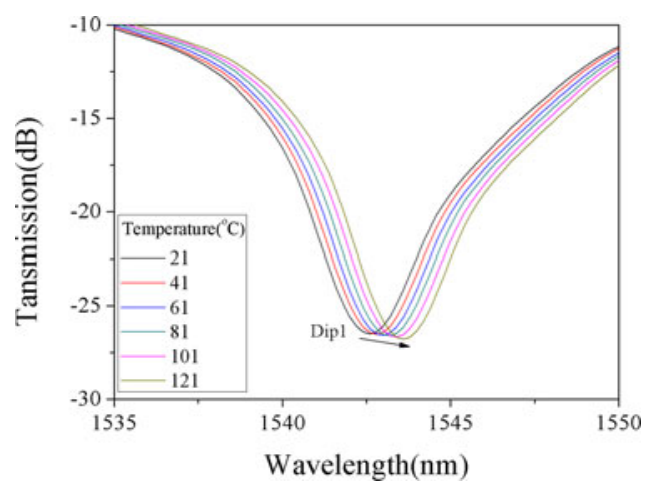

Fig. 12. Transmission spectrum response shift for different temperature.

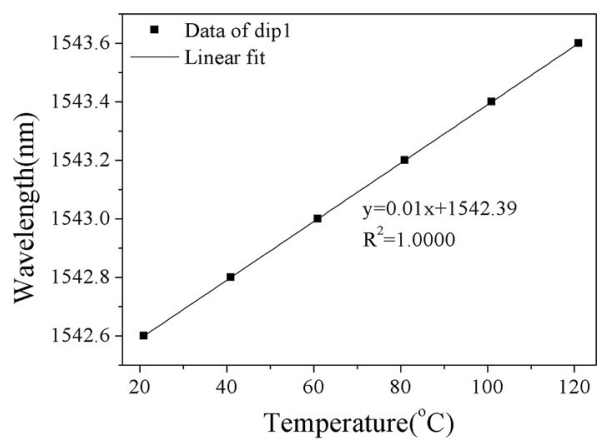

(a)

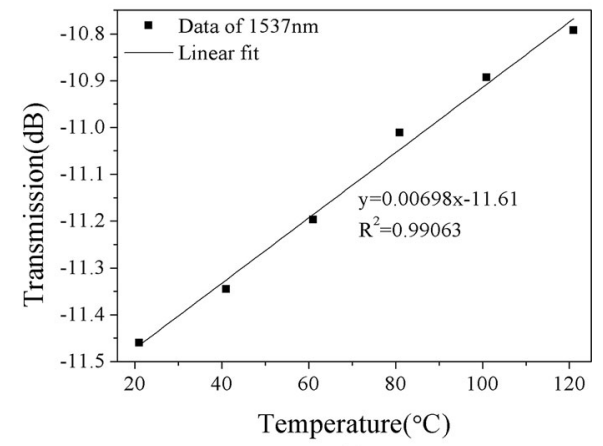

(b)

Fig. 13. (a) Dip wavelength shifts as a function of temperature (b) Transmission as a function of temperature (at $1537 \mathrm{~nm}$ ).

$21{ }^{\circ} \mathrm{C}$ (room temperature) without temperature variation as a starting point. A super-continuum source was used for launching power into the STMS device and the wavelength shifts were monitored by the optical spectrum analyzer as described above.

The measured spectral responses are shown in Fig. 12, it is clearly shown that when the surrounding temperature increases a red shift of the wavelength dip occurs accompanied by a decline of transmission.

Fig. 13 shows the measured temperature dependence for the hybrid STMS device over a temperature range from $21^{\circ} \mathrm{C}$ to $121^{\circ} \mathrm{C}$ at intervals of $20^{\circ} \mathrm{C}$. In Fig. $13(\mathrm{a})$, the mode interference dip exhibits very small shifts to longer wavelengths (red shift) when the temperature was increased from $21^{\circ} \mathrm{C}$ to $121{ }^{\circ} \mathrm{C}$. The temperature sensitivity was determined to be $0.01 \mathrm{~nm} /{ }^{\circ} \mathrm{C}$ over the temperature range concerned. Fig. 13(b) shows the 
intensity-temperature sensitivity is $0.007 \mathrm{~dB} /{ }^{\circ} \mathrm{C}$ at the single wavelength of $1537 \mathrm{~nm}$. Both data sets show excellent linearity, demonstrated by a linear regression value of $\mathrm{R}^{2}=$ 1.0000 and 0.9906. As expected, the measured thermal sensitivity of the STMS device has been proven to be very low, confirmed by the thermal sensitivity obtained in the experiment for the hybrid STMS device which is circa $10 \mathrm{pm} /{ }^{\circ} \mathrm{C}$. Since the dip red-shifts increase monotonically as a function of temperature, it is possible to apply a simple correction factor to mitigate the temperature induced errors.

\section{CONCLUSION}

In this paper, a curvature sensor based on STMS fibre structure has been investigated theoretically and experimentally. The measured transmission spectra demonstrate a general agreement with the theoretical predictions. This sensor can be interrogated using an optical spectral analyzer (OSA) or a ratiometric interrogation system as developed previously. Using the novel sensor design developed in this investigation a higher average curvature sensitivity of $-2.42 \mathrm{~nm} / \mathrm{m}^{-1}$ over a curvature measurement range of $0-1.7390 \mathrm{~m}^{-1}$ and $-7.09 \mathrm{~dB} / \mathrm{m}^{-1}$ at an operating wavelength of $1537 \mathrm{~nm}$ has been achieved, compared with a conventional SMS fibre structure sensor. The temperature dependence of this optical fibre curvature sensor has also been demonstrated, the temperature sensitivities are $0.01 \mathrm{~nm} /{ }^{\circ} \mathrm{C}$ over a wavelength range of $1535-1550 \mathrm{~nm}$ and circa $0.007 \mathrm{~dB} /{ }^{\circ} \mathrm{C}$ at the wavelength of $1537 \mathrm{~nm}$, over a temperature range of $21-121^{\circ} \mathrm{C}$. Further optimization of this hybrid fibre sensor geometry, such as twisting turns and twisting positions, will result in a more compact fibre optic sensor device with improved performance.

\section{REFERENCES}

[1] Q. Wang, G. Farrell, and W. Yan, "Investigation on single-modemultimode-single-mode fiber structure," J. Lightw. Technol., vol. 26, no. 5, pp. 512-519, Mar. 2008.

[2] Q. Wang and G. Farrell, "Multimode-fiber-based edge filter for optical wavelength measurement application and its design," Microw. Opt. Technol. Lett., vol. 48, no. 5, pp. 900-902, 2006.

[3] A. M. Hatta et al., "Polarization dependence of an edge filter based on singlemode-multimode-singlemode fibre," Opt. Laser Technol., vol. 42 , no. 6, pp. 1044-1048, 2010.

[4] W. S. Mohammed, P. W. Smith, and X. Gu, "All-fiber multimode interference bandpass filter," Opt. Lett., vol. 31, no. 17, pp. 2547-2549, 2006.

[5] W. S. Mohammed, A. Mehta, and E. G. Johnson, "Wavelength tunable fiber lens based on multimode interference," J. Lightw. Technol., vol. 22, no. 2, pp. 469-477, Feb. 2004.

[6] Q. Wu et al., "Single-mode-multimode-single-mode fiber structures for simultaneous measurement of strain and temperature," Microw. Opt. Technol. Lett., vol. 53, no. 9, pp. 2181-2185, 2011.

[7] D.-P. Zhou, L. Wei, W.-K. Liu, and J. W. Y. Lit, "Simultaneous strain and temperature measurement with fiber bragg grating and multimode fibers using an intensity-based interrogation method," IEEE Photon. Technol. Lett., vol. 21, no. 7, pp. 468-470, Apr. 2009.

[8] S. Silva et al., "Temperature and strain-independent curvature sensor based on a singlemode/multimode fiber optic structure," Meas. Sci. Technol., vol. 22, no. 8, 2011, Art. no. 085201.

[9] Q. Wu et al., "High sensitivity SMS fiber structure based refractometeranalysis and experiment," Opt. Express, vol. 19, no. 9, pp. 7937-7944, 2011.

[10] P. Wang et al., "High-sensitivity, evanescent field refractometric sensor based on a tapered, multimode fiber interference," Opt. Lett., vol. 36 , no. 12, pp. 2233-2235, 2011.
[11] Q. Wu et al., "Fiber refractometer based on a fiber Bragg grating and single-mode-multimode-single-mode fiber structure," Opt. Lett., vol. 36, no. 12, pp. 2197-2199, 2011

[12] Y. Zhao et al., "Liquid concentration measurement based on SMS fiber sensor with temperature compensation using an FBG," Sensors Actuators B, Chem., vol. 196, pp. 518-524, 2014.

[13] S. W. James and R. P. Tatam, "Optical fibre long-period grating sensors: Characteristics and application," Meas. Sci. Technol., vol. 14, no. 5, pp. R49-R61, 2003.

[14] O. Frazão et al., "All-fiber Mach-Zehnder curvature sensor based on multimode interference combined with a long-period grating," Opt. Lett., vol. 32, no. 21, pp. 3074-3076, 2007.

[15] M. Gander et al., "Bend measurement using Bragg gratings in multicore fibre," Electron. Lett., vol. 36, no. 2, p. 1-2, 2000.

[16] C. Gouveia, P. Jorge, J. Baptista, and O. Frazao, "Temperatureindependent curvature sensor using FBG cladding modes based on a core misaligned splice," IEEE Photon. Technol. Lett., vol. 23, no. 12, pp. 804-806, Jun. 2011.

[17] L. Mao et al., "Highly sensitive curvature sensor based on single-mode fiber using core-offset splicing," Opt. Laser Technol., vol. 57, pp. 39-43, 2014.

[18] Y. Gong, T. Zhao, Y.-J. Rao, and Y. Wu, "All-fiber curvature sensor based on multimode interference," IEEE Photon. Technol. Lett., vol. 23, no. 11, pp. 679-681, Jun. 2011.

[19] Y. Zhao, L. Cai, and H.-F. Hu, "Fiber-optic refractive index sensor based on multi-tapered SMS fiber structure," IEEE Sensors J., vol. 15, no. 11, pp. 6348-6353, Nov. 2015

[20] L. Palmieri and A. Galtarossa, "Twist effects in multi-mode fibers," in Proc. IEEE 3rd Mediterranean Photon. Conf., 2014, pp. 1-3.

[21] K.-P. Ho and J. M. Kahn, "Statistics of group delays in multimode fiber with strong mode coupling," J. lightw. technol., vol. 29, no. 21, pp. 3119-3128, Nov. 2011.

[22] C. Antonelli et al., "Stokes-space analysis of modal dispersion in fibers with multiple mode transmission," Opt. exp., vol. 20, no. 11 , pp. 11718-11733, 2012.

[23] L. Palmieri, "Coupling mechanism in multimode fibers," in Proc. SPIE OPTO, Int. Soc. Opt. Photon., 2013, p. 90090G-1-90090G-9, 2013

Ke Tian received the B.S. degree in physics from Heilongjiang University, Harbin, China, in 2014. He is currently working toward the Ph.D degree in optical engineering at Harbin Engineering University, Harbin. His research interests include fiber-optic sensors and devices.

Yifan Xin received the B.S. degree in communication engineering from Harbin Engineering University (HEU) in June 2015. She began her career as a graduate student in the College of Science, HEU, in August 2016. Her research interests include compound glass materials and fiber-optic sensor and currently focused on rare earth doped $\mathrm{SiO}_{2}$ fiber sensor.

Wenlei Yang received the $\mathrm{Ph}$. D. degree in photonics from Harbin Engineering University, Harbin, China, in 2016. She is currently a Postdoctoral at the Key Lab of In-fiber Integrated Optics, Ministry Education of China, Harbin Engineering University. Her research interests include fiber-optic sensing and long-period fiber grating.

Tao Geng received the B.S. and Ph.D. degrees in precision instrument and mechanology from Harbin Institute of Technology, Heilongjiang, China, in 1995 and 2003, respectively, and the M.Eng. degree in optoelectronics from Changchun Optics Precision Mechanical School, Jilin, China, in 1998. He was a postdoctoral in the School of Aeronautics, Harbin Institute of Technology, Heilongjiang, from 2003 to 2005 . He is currently an Associate Professor in the Key Lab of In-fiber Integrated Optics, Ministry Education of China, Harbin Engineering University, China. His research interests include fiber micromachining and long-period fiber grating. 
Jing Ren born in 1981. He received the Doctoral degree in materials science in 2009 from the University of Pardubice, Pardubice, Czech. In 2015, he joined the Key Lab of In-fiber Integrated Optics, Ministry Education of China, Harbin Engineering University, China, and became a supervisor for Ph.D. candidates. He has published more than 80 peer-reviewed papers in Applied Physics Letters, Optics Letters, Optics Express, The Journal of Physical Chemistry C, and the Journal of the American Ceramic Society. He was in charge of or participated in more than eight projects, including two projects supported by the National Natural Science Foundation of China, two projects by China Postdoctoral Science Foundation, and one project by Research Funds for Young Teachers, Ministry of Education of China. His research interest include design and characterization of novel inorganic functional materials, such as heavy metal and chalcogenide glasses and glass-ceramics, phosphors and films, exploration of their potential applications in sensing, imaging, solar cells, and integrated IR optics.

Ya-Xian Fan was born in the Inner Mongolia, China, in 1975. She received the B.S. and Ph.D. degrees in optics from the Department of Physics, Nankai University, Tianjin, China, in 1998 and 2003, respectively.

From 2003 to 2005, she was a Postdoctoral Fellow with the Department of Physics, also with the National Laboratory of Solid State Microstructures, Nanjing University. From 2005 to 2011, she was an Associate Professor with the Department of Physics, Nanjing University. Since 2011, she has been a Professor with the College of Science, Harbin Engineering University, HarbinChina. She is the author of more than 100 articles and more than 20 inventions. Her research interests include microstructure optical waveguides, mode field regulations, terahertz waveguide devices, microstructure fiber photonics, all-optical logical devices, and nonlinear signal processing.

Prof. Fan was awarded New Century Excellent Talents in the University of Ministry of Education of China in 2008. She is a Senior Member of the Optical Society of China.

Gerald Farrell completed an electronic engineering degree from University College Dublin in 1979, he spent a number of years as a design engineer developing optical fiber transmission systems before joining the Dublin Institute of Technology (DIT). He received his Ph.D. from Trinity College Dublin for research in all-optical synchronization using self-pulsating laser diodes. Between 1997 and 2003 he was a director of the startup company PX Instrument Technology, focusing on optical fiber system test and measurement systems.

Prof. Farrell is the founder and Director of the DIT Photonics Research Centre (PRC). He leads a multinational research team of doctoral, postdoctoral and senior researchers focusing on several areas of optical fiber sensing research including photonic crystal fiber sensors for environmental and energy systems sensing, novel sensors for composite materials and medical devices, LC infiltrated PCF sensors and micro-fiber and nanowire sensors for chemical and bio-sensing. Prof. Farrell has also led the development of research collaborations with a number of research groups in China, Poland and elsewhere. He has over 320 publications in the area of photonics.

Prof. Farrell is also a member of the Optical Society of America and an advisory professor in China at Beijing University of Posts and Telecommunications and at Harbin Engineering University. He is also Dean of the College of Engineering and Built Environment at the DIT.
Elfed Lewis (SM'00) received the B.Eng. (Hons.) degree in electrical and electronic engineering and the Ph.D. degree, both from Liverpool University, Liverpool, U.K., in 1978 and 1987, respectively. He is an Associate Professor and the Director of the Optical Fibre Sensors Research Centre at University of Limerick, which he founded in 1996. He is Fellow of the Institute of Physics and IET. He has authored and coauthored more than 70 journal papers and made in excess of 300 contributions to international conferences. He currently holds seven patents on optical fiber sensor devices. In 2005, he received the University of Limerick Special Achievement in Research Award and was a Fulbright Scholar with CREOL (University of Central Florida) in 2008. He was a Distinguished Lecturer for the IEEE Sensors Council for the period July 2013-June 2015 and the General Chair of the recent European Workshop on Optical Fibre Sensors held at the University of Limerick, Ireland.

Pengfei Wang received the Ph.D. degree in optics engineering from the Photonics Research Centre (PRC), Dublin Institute of Technology (DIT), Dublin, Ireland, in November 2008. He worked for the European Space Agency and the Italian Space Agency as a Research Assistant at the Institute of Microelectronics and Microsystems, Italian National Research Council in Bologna, Italy, sponsored by both the Italian Ministry of Foreign Affairs and the United Nations Educational, Scientific and Cultural Organization from 2004 to 2005. He worked as a Research Associate in the Photonics Research Centre, DIT, Dublin Ireland, supported by the Irish Research Council Government Postdoctoral Scholarship from 2009 to 2010. In 2010, he joined the Optoelectronics Research Centre, University of Southampton, funded by the EU Marie Curie Research Fellowship. In 2011, he joined the Advanced Laser Lab of the SPI lasers (Southampton) as a part-time Research Fellow. He has been appointed as a Senior Research Fellow (Tenured) at the PRC of the DIT from 2013 to 2015. Since September 2015, he has been working with the College of Science, Harbin Engineering University, China, as a full-time Distinguished Professor, sponsored by the Chinese government programme entitled "The Recruitment Program of Global Youth Experts." His research interests include compound glass materials, fiber lasers, computational photonics (modeling, simulation, and optimization), photonic devices (such as microfiber/nanowire-based photonic devices, fiber optic sensors, photonic integrated circuits, liquid crystal based photonic devices, laser micromachining, etc.) and applications development (optical communication and optical sensing). He has authored and coauthored more than 160 papers in academic journals and international conferences so far. 